\title{
Phenotypic Changes in Hepatic Stellate Cells in Response to Toxic Liver Injury
}

\author{
Bryan L. Copple
}

Published online: 17 September 2014

(c) Springer Science+Business Media New York 2014

\begin{abstract}
Hepatic stellate cells (HSCs) are the main extracellular matrix-producing cell type in the liver. These cells exist in a quiescent state in normal liver and an activated state in damaged liver. During quiescence, HSCs store and release various retinoids. After injury to the liver, however, various protein and non-protein mediators are released from cells that stimulate HSCs to differentiate into myofibroblasts, a process termed activation. Activated HSCs begin to express $\alpha$-smooth muscle actin and migrate to sites of injury through chemotaxis. Within the damaged region of liver, these cells become contractile and produce growth factors that promote hepatocyte replication and angiogenesis, produce extracellular matrix that forms the scaffold for liver repair, and produce proteins that modulate immune cell function. Once liver repair is complete and liver function is restored, HSCs revert back to a quiescent phenotype or die by apoptosis. If liver injury becomes chronic, however, these processes persist resulting in the formation of an extensive scar (i.e., fibrosis) that ultimately leads to liver failure. Because of the importance of these cells to the development of liver fibrosis, there has been extensive research into understanding the mechanisms that drive HSC activation after injury and the mechanisms that regulate reversion of these cells back to quiescence after resolution of injury. In addition, there has been great interest in identifying the various functions of HSCs after acute and chronic injury. The aim of this review is to briefly discuss the phenotypic changes that occur in HSCs after acute and chronic liver injury and to highlight some of
\end{abstract}

\section{B. L. Copple $(\square)$}

Department of Pharmacology and Toxicology, Michigan State University, B403 Life Sciences Building, East Lansing,

MI 48824, USA

e-mail: copple@msu.edu the important functions of these cells during repair of the liver.

Keywords Hepatic stellate cells · Liver - Fibrosis · Regeneration · Inflammation

\section{Introduction}

After many years of research, Friedman and colleagues identified hepatic stellate cells as the principle extracellular matrix-producing cell in the liver, and subsequent studies have confirmed their importance in the development of liver fibrosis [1]. Although other cells have been proposed to contribute to collagen deposition during chronic injury, such as hepatocytes and bile duct epithelial cells through epithelial to mesenchymal transition, bone marrow-derived fibrocytes, and portal fibroblasts, recent studies have solidified HSCs as the predominant collagen-producing cell in the liver during injury $[2 \cdot 3 \bullet \cdot \bullet$.

HSCs are located between the sinusoidal endothelium and hepatocytes in the Space of Disse. These cells exist in two main phenotypes termed quiescent and activated. During quiescence, HSCs store vitamin A and play an important role in vitamin A homeostasis. During liver injury, however, a number of protein and non-protein mediators are produced that stimulate HSCs to differentiate into myofibroblasts, a process termed activation [4]. Once activated, these cells migrate to sites of injury, proliferate, produce collagen, and regulate immune cell function by producing various immunomodulatory proteins. After acute liver injury, secretion of extracellular matrix by HSCs forms the initial scar, which may protect parenchymal cells from further damage and provide the initial scaffold for repair. Once repair is completed, HSCs revert 
back to a quiescent state or die by apoptosis. During chronic injury, HSC activation and collagen deposition persists resulting in the formation of an extensive scar (i.e., fibrosis) that ultimately impairs liver function. Because these cells are crucial to the development of liver fibrosis, a disease for which there remains no effective therapy, there has been extensive research into understanding the mechanisms that drive HSC activation and the mechanisms that regulate reversion of these cells back to quiescence. In addition, there has been great interest in identifying HSC functions after injury. Surprisingly, although these cells were initially described over a century ago, the list of HSC functions continues to grow today with the advent of new methods to study these cells in vivo. The aim of this review is to discuss the phenotype and function of HSCs after acute and chronic liver injury. It will begin with a discussion of HSC function and phenotype during chronic injury, and end with a discussion of HSC phenotype and function during acute injury, where exciting, new functions of HSCs have recently been uncovered.

\section{Activation and Function of HSCs During Chronic Liver Injury}

During chronic liver injury, cells in the liver, such as hepatocytes, macrophages, and others, begin to secrete a variety of proteins that stimulate HSCs to differentiate into myofibroblasts, a process termed "activation." These mediators also render HSCs more responsive to paracrine and autocrine growth factors that stimulate HSCs to proliferate and secrete collagen, and stimulate HSC contraction. Furthermore, cytokines are produced that stimulate activated myofibroblasts to migrate to injured regions of liver where they deposit collagen that ultimately forms the scar. The mediators that govern these processes during chronic injury are briefly described in the following sections.

\section{Mediators that Stimulate HSC Proliferation}

Several mediators have been shown to stimulate HSC proliferation, including growth factors, such as PDGFs and FGFs, neurotransmitters, such as norepinephrine and serotonin, and the protease thrombin. Within this group of HSC mitogens, PDGF-B is the most potent.

Treatment of activated HSCs in vitro with PDGF-B stimulates proliferation [5]. This requires prior HSC activation, because this process upregulates PDGF receptor $\beta$ on HSCs [6]. Similarly, activation of HSCs in vivo by chronic treatment with carbon tetrachloride increases expression of PDGF receptor $\beta$ on these cells [6]. Activation of the PDGF receptor stimulates signaling through various pathways in
HSCs that contribute to proliferation, including protein kinase C, Erk1/2, and phospatidylinositol-3 kinase [7]. In support of a role for PDGF-B in the development of liver fibrosis, transgenic overexpression of PDGF-B stimulates development of liver fibrosis [8]. Similarly, transgenic expression of PDGF-A in mice promotes liver fibrosis [9]. Compared to PDGF-B, however, PDGF-A is far less potent at simulating HSC proliferation in vitro. Accordingly, it is unclear whether PDGF-A achieves concentrations during chronic injury that affect HSC function. PDGF-C is the least potent stimulator of HSC proliferation, and studies have shown that liver fibrosis is not affected in PDGF-C knockout mice $[10,11]$. However, similar to PDGF-A transgenic mice, transgenic expression of PDGF-C in mice produces liver fibrosis [12]. Whether the levels of PDGF-C achieved in this manner are pathologically relevant, however is not known. PDGF-D is also upregulated in animal models of fibrosis, and stimulates HSC proliferation in vitro with similar potency to that of PDGF-B. It still remains to be determined whether PDGF-D contributes to the development of liver fibrosis in animal models or humans.

Studies have shown that FGF-2 stimulates HSC proliferation in vitro, and that the mitogenic effects of TGF- $\beta 1$ in vitro may be mediated by the release of FGF-2 from HSCs [13]. Liver fibrosis, induced by chronic carbon tetrachloride treatment, is reduced in FGF-1 and FGF-2 double-knockout mice [14]. Interestingly, similar numbers of $\alpha$-smooth muscle actin-positive cells were observed in wild-type and FGF-1 and FGF-2 double-knockout mice, suggesting that FGF-1 and FGF-2 may stimulate collagen production by HSCs but not proliferation in vivo [14]. It is possible though that FGF-2 may be an important HSC mitogen in other models of liver fibrosis, although this remains to be determined.

Thrombin is most well-known for its ability to stimulate fibrin deposition after injury by cleaving fibrin and other factors in the coagulation cascade. Thrombin can also activate signal transduction pathways in cells by activating protease-activated receptor-1 (PAR-1) [15]. This receptor is expressed on HSCs, and studies have shown that HSCs proliferate in vitro when treated with thrombin, an effect that was prevented in HSCs from PAR-1 knockout mice [16]. In addition, liver fibrosis is reduced in PAR-1 knockout mice and in mice treated with a PAR-1 antagonist $[17,18]$. Interestingly, recent studies showed that fibrin is deposited in the livers of patients with fibrosis indicating that even though coagulation factors may be depleted in patients with diminished liver function, sufficient liver function is present to activate the coagulation system locally in the liver, which may stimulate HSC proliferation in a PAR-1-dependent manner [19].

HSCs express many neural proteins, including neurotrophins and their receptors, glial fibrillary acidic protein, 
and many others [20], and studies have shown that exposure of HSCs to various neurotransmitters stimulates HSC proliferation. Serotonin enhances PDGF-induced proliferation in vitro [21]. Norepinephrine is mitogenic for HSCs in vitro, an effect which is enhanced by co-stimulation with neuropeptide Y [22]. In support of a role for norepinephrine in the development of fibrosis, Dbh knockout mice, which are deficient in norepinephrine, showed reduced liver fibrosis [23]. Similar to norepinephrine, the neurotransmitter acetylcholine is an HSC mitogen in vitro [24]. Taken together, these studies suggest that neural input to the liver may regulate HSC proliferation and ultimately liver fibrosis. The importance of this process in patients with liver fibrosis, however, remains to be investigated.

Mediators that Stimulate Production of Extracellular Matrix by HSCs

The growth factor TGF- $\beta$ has a number of functions, including regulation of the immune system and regulation of collagen deposition during tissue repair [25]. TGF- $\beta$ stimulates collagen synthesis by activated HSCs, and increased levels of TGF- $\beta$ are detected in humans and animals with fibrosis [26]. TGF- $\beta$ is secreted in a latent form by Kupffer cells and HSCs during liver injury. Once secreted, TGF- $\beta$ is converted to its active form by thrombospondin-1 or plasmin or by interaction with the integrin, $\alpha \mathrm{V} \beta 6[25,27]$. TGF- $\beta$ activates the Smad transcription factor pathway and mitogen-activated protein kinases in HSCs which increases expression of collagen [28, 29]. Activation of these pathways in HSCs by TGF- $\beta$ increases expression of procollagen. In mice, overexpression of TGF- $\beta$ by an adenovirus increased collagen in the liver [30]. Furthermore, liver fibrosis was reduced in TGF- $\beta$ knockout mice confirming that this growth factor is essential for collagen deposition in vivo during chronic injury [30].

Another mechanism by which TGF- $\beta$ may stimulate collagen production in the liver during chronic injury is through the upregulation of connective tissue growth factor (CTGF). Studies have demonstrated increased levels of CTGF mRNA in patients with primary biliary cirrhosis, primary sclerosing cholangitis, biliary atresia, and in patients infected with hepatitis B [31-34]. Similarly, CTGF mRNA is upregulated in the livers of rodents treated chronically with carbon tetrachloride or subjected to bile duct ligation [35]. In fibrotic livers, CTGF is predominantly expressed in HSCs and bile duct epithelial cells. In cultured HSCs, CTGF expression is increased in activated HSCs and in HSCs treated with TGF- $\beta$ [36]. PDGF also stimulates the production of CTGF by HSCs, an effect that is blocked by neutralization of TGF- $\beta$ [36]. Treatment of HSCs with CTGF stimulates collagen production and HSC proliferation and migration [36]. In support of a role for CTGF in the development of liver fibrosis, inhibition of CTGF in vivo with an siRNA decreases collagen levels [37].

In addition to growth factors, recent studies have shown that the proinflammatory cytokine, interleukin-17A (IL17A), stimulates HSCs to produce collagen. In these studies, treatment of HSCs in vitro with IL-17A increased expression of collagen- $\alpha 1$ (I) in a Stat3-dependent manner [38]. In support of a role for this cytokine in the development of liver fibrosis, collagen deposition was reduced in IL-17RA knockout mice after bile duct ligation or chronic carbon tetrachloride treatment. Considering that IL-17A has also been linked to tissue damage from autoimmune diseases, targeting IL-17A in autoimmune liver diseases, such as primary biliary cirrhosis, may be an effective way to reduce not only liver injury, but also fibrosis.

In addition to the protein mediators described above, studies indicate that products of oxidative stress stimulate HSCs to produce collagen. Schneiderhan et al. showed that oxidized low-density lipoproteins (LDL), which are increased in baboons fed ethanol and in alcoholic patients, stimulated collagen production by HSCs in a CD36dependent manner [39]. In addition, these studies showed that CD36 is upregulated on activated HSCs, and that CD36 is expressed on HSCs in the livers of alcoholic patients. Similar to oxidized LDL, products of lipid peroxidation, such as 4-hydroxy-2,3-nonenal, stimulate HSCs to produce collagen [40]. Collectively, these studies suggest that oxidative stress, potentially from immune sources or xenobiotics, may stimulate collagen production by activated HSCs.

\section{Mediators that Stimulate HSC Chemotaxis}

After injury, HSCs migrate to damaged regions of liver. Chemokines, released at the site of injury, stimulate HSC migration by forming a chemotactic gradient. This process is similar to immune cell chemotaxis, and many of the chemokines that stimulate migration of immune cells also stimulate HSC chemotaxis, such as MIP- $1 \alpha$, MIP- $1 \beta$, RANTES, and MCP-1 [41-43]. In addition to these traditional chemokines, various growth factors, such as VEGF and PDGF, and the protein osteopontin stimulate HSC chemotaxis [44-46].

HSCs express the traditional chemokine receptors, Ccr2 and $\mathrm{Ccr} 5$, which are activated by MIP- $1 \alpha$, MIP- $1 \beta$, RANTES, and MCP-1 [42, 43]. In vitro, HSCs migrate toward these chemokines in a Ccr2- or Ccr5-dependent mechanism, and in vivo, liver fibrosis is reduced in $\mathrm{Ccr} 2$ and $\mathrm{Ccr} 5$ knockout mice $[42,43]$. To distinguish between the role of these receptors on immune cells and non-immune cells, bone marrow from wild-type mice was transplanted into 
Ccr2 and Ccr5 knockout mice in these studies. Although the chemokine receptors remained functional on immune cells in these mice, liver fibrosis was decreased, suggesting that activation of these receptors on a non-immune cell type was profibrotic. Since these receptors are expressed by HSCs, it was proposed by the authors that activation of these receptors on HSCs promoted fibrosis.

Osteopontin is another chemokine that is chemotactic for HSCs [46]. The receptor for this protein is $\alpha_{v} \beta_{3}$ integrin, which is expressed by HSCs [47]. Conflicting results exist, however, with regard to the role of this chemokine in the development of liver fibrosis. Whereas liver fibrosis was reduced in osteopontin knockout mice fed a methionine/ choline-deficient diet to model nonalcoholic steatohepatitis, liver fibrosis was not reduced in mice subjected to bile duct ligation [48, 49]. In addition, treatment of these mice chronically with carbon tetrachloride resulted in enhanced liver fibrosis, which may have resulted from increased liver injury in these mice [50]. In a separate study, inhibition of osteopontin in mice with a neutralizing antibody decreased fibrosis in mice fed a methionine/choline-deficient diet and in mice treated chronically with carbon tetrachloride [51]. It is not completely clear why these studies are conflicting, although the difference may have resulted from the method of osteopontin inhibition (i.e., knockout mouse vs. neutralization). Lastly, the growth factors, VEGF and PDGF, have been shown to be chemotactic for HSCs in vitro, although their importance in vivo remains to be investigated [44, 45].

\section{Mediators that Stimulate HSC Contraction}

A key step in the wound-healing process is a contraction of the wound to facilitate closer. This task is performed by myofibroblasts in the skin and in other tissues. In the liver, activated HSCs contract when exposed to various vasoactive mediators. Contraction of HSCs has been shown to modulate sinusoidal blood flow, and it has been proposed that this process in fibrotic livers may lead to increased portal resistance and ultimately portal hypertension [52-54]. Endothelin-1 is the most well-characterized mediator of HSC contraction. HSCs express endothelin-1 receptors, and these cells contract when exposed to endothelin-1 [55, 56]. In addition, endothelin-1 levels are increased in cirrhotic human livers and studies suggest that endothelin-1 antagonists, such as bosentan, are useful for the treatment of portopulmonary hypertension in patients with cirrhosis [57-59].

\section{Activation and Function of HSCs During Acute Liver Injury}

Similar to chronic liver injury, HSCs are activated in the liver during acute liver injury. In rodents, levels of $\alpha$-SMA and type I collagen, hallmarks of HSC activation, are increased in centrilobular lesions after a single dose of carbon tetrachloride [60]. Similarly in humans suffering from acute liver failure from viral hepatitis, drug toxicity, or autoimmune hepatitis, there is an increase in $\alpha$-SMAexpressing cells within necrotic lesions indicating activation of HSCs [61]. While persistent HSC activation is detrimental to liver function during chronic liver injury, activation of HSCs during acute liver injury may benefit liver function by producing pro-reparative growth factors, by depositing extracellular matrix that provides the scaffold for repair, and by modulating immune cell function. The role of HSCs in these processes during acute liver injury is described in brief below.

\section{Role of HSCs in Liver Regeneration}

Much of our knowledge regarding HSC function during liver regeneration has been discovered in rodents subjected to partial hepatectomy. Although this model does not typically involve substantial acute liver injury, information gained from partial hepatectomy studies may be applicable to liver repair after acute liver injury; however, this remains to be confirmed. A recent study utilizing gliotoxin demonstrated a potential role for HSCs in early cell proliferation after partial hepatectomy and a role in termination of liver regeneration [62•]. Gliotoxin is a fungal metabolite that reduces fibrosis in animal models by stimulating HSC apoptosis [63]. This chemical has been used to investigate HSC function in the liver, although gliotoxin can affect other liver cell types, including Kupffer cells [64]. Interestingly, though, pretreatment of rats with gliotoxin substantially reduced early cell proliferation after partial hepatectomy indicating a potential role for HSCs in liver regeneration [62•]. This was also associated with a decrease in hepatocyte growth factor (HGF) levels. Previous studies have shown that HSCs synthesize HGF, although interestingly, after partial hepatectomy, it was only unactivated HSCs that produced HGF [65]. Another mechanism by which HSCs may contribute to cell proliferation is through expression of TGF- $\alpha$. TGF- $\alpha$ stimulates hepatocyte proliferation through an autocrine mechanism, and studies have shown that TGF$\alpha$ levels are increased after partial hepatectomy [66]. In addition to hepatocytes, another cell type that expresses TGF- $\alpha$ is HSCs, which may facilitate regeneration through the release of this factor [67]. It was also recently shown that HSCs may promote liver regeneration through delta-like 1 homolog (DLK-1) [68]. DLK-1 is a transmembrane protein that is cleaved by ADAM-17 which releases the extracellular domain of DLK-1 [69]. This domain activates signal transduction pathways in cells by mechanisms that are not fully understood. A recent study showed that after partial hepatectomy, DLK-1 levels are increased in HSCs [68]. 
Furthermore, in this study, co-culture of HSCs isolated from mice subjected to partial hepatectomy with hepatocytes stimulated hepatocyte proliferation, an effect that was inhibited by a DLK-1-neutralizing antibody. Similarly, inhibition of DLK-1 in partial hepatectomized mice reduced cell proliferation indicating a key role for this protein in regeneration of the liver.

Another mechanism by which HSCs may facilitate liver regeneration after injury is through the regulation of angiogenesis, the process of new blood vessel formation. Key factors in this process include VEGF, angiopoietin-1 (Ang-1), and Ang-2. Angiogenesis is important for regeneration of the liver after partial hepatectomy, as studies have shown that neutralization of VEGF reduces sinusoidal endothelial cell proliferation and impairs liver repair [70]. During angiogenesis, hepatocyte nodules are invaded by sinusoidal endothelial cells to reform the sinusoidal vasculature during regeneration [71]. The importance of HSCs to this process is not fully understood, however, studies have shown HSCs express Ang-1 and Ang-2 after partial hepatectomy [72]. These two factors are important for remodeling and maturation of vessels, and it is possible that the release of these proteins from HSCs contributes to this process. In addition, deposition of extracellular matrix by HSCs may facilitate angiogenesis. Studies have shown that after partial hepatectomy, clusters of hepatocytes are first invaded by activated HSCs which deposit matrix [71]. This process is soon followed by the invasion of sinusoidal endothelial cells into the hepatocytes clusters

In addition to stimulating cell proliferation during repair, HSCs may be important for termination of cell proliferation once liver repair has completed. In support of this, treatment of rats with gliotoxin at 5 days after partial hepatectomy resulted in increased cell proliferation at 7 days, suggesting that termination of liver regeneration was affected [62•]. Associated with this was a decrease in type I collagen protein in the liver and a decrease in TGF$\beta$. The authors proposed that the increase in hepatocyte proliferation may have resulted from a decrease in hepatocyte-extracellular matrix interactions which can increase hepatocyte proliferation, or a decrease in TGF- $\beta$ which may be important for termination of hepatocyte proliferation $[73,74]$. Once liver repair is completed, HSCs may also contribute to the maturation of newly formed hepatocytes through the release of epimorphin. Levels of epimorphin are increased in HSCs after partial hepatectomy, and studies have shown that epimorphin may increase differentiated features of hepatocytes in culture [75]. Whether this process is important for liver regeneration, however, remains to be determined.

While the above studies suggest that HSCs could play a key role in coordinating liver repair after injury by producing growth factors and extracellular matrix that regulate cell proliferation and migration, these findings require confirmation using more selective methods to inhibit HSC function during liver regeneration.

\section{Potential Role of HSC-Macrophage Interactions} in Regulation of Acute Liver Injury

With the recent development of methods to selectively deplete HSCs in the liver, previously unrecognized functions of HSCs have been identified. One finding that was quite surprising was the ability of HSCs to modulate liver injury. The first study to demonstrate this used transgenic mice in which the herpes simplex thymidine kinase gene was driven by the GFAP promoter [3]. Expression of this gene in HSCs makes them susceptible to killing by ganciclovir allowing for depletion of HSCs in mice. While these studies were conducted to confirm the importance of HSCs in collagen deposition during fibrosis, an unexpected finding was that the depletion of HSCs decreased liver injury after treatment with carbon tetrachloride and allyl alcohol [3]. This was also associated with an increase in the levels of the anti-inflammatory cytokine, interleukin-10, suggesting that the depletion of HSCs renders the liver less inflammatory during acute liver injury. Using the same system to deplete HSCs, another group showed recently that HSCs are important for liver injury after bacterial lipopolysaccharide (LPS) treatment and after ischemia/ reperfusion [76•]. In these studies, depletion of HSCs decreased mRNA levels of tumor necrosis factor- $\alpha$ and CXCL1, and decreased hepatic neutrophil accumulation and liver injury in these two models of inflammatory liver injury. The authors proposed that HSCs are an important source of TNF- $\alpha$ and CXCL1, and that the depletion of HSCs reduced the pool of these cytokines which are important for neutrophil-dependent liver injury in these models. While this explanation is quite plausible, an alternative explanation may be that HSCs modulate macrophage function during acute liver injury resulting in an altered production of cytokines by these cells. Two recent studies lend support for this possibility. Chang and colleagues showed that exposure of macrophages to conditioned medium from activated HSCs increased IL- 6 and IL-8 and decreased IL-10, indicating that activated HSCs polarize macrophages toward a proinflammatory phenotype [77••]. In a second study from our laboratory, selective deletion of the transcription factor, hypoxia-inducible factor- $1 \alpha$, in hepatic stellate cells in the liver prevented upregulation of the inflammatory cytokines TNF- $\alpha$, Cxcl1, $\mathrm{Cxcl} 2, \mathrm{Ccl} 3$, and $\mathrm{Ccl} 4$ and decreased the percentage of $\mathrm{Gr} 1^{\mathrm{hi}} \mathrm{CD} 11 \mathrm{~b}^{\mathrm{hi}} \mathrm{F} 4 / 80^{+}$proinflammatory macrophages in the liver after treatment with a single dose of carbon tetrachloride [78]. Considering that these end-points are markers of proinflammatory macrophages, this study 
indicated that HSCs polarize macrophages toward a proinflammatory phenotype during acute liver injury. Taken together, these four studies suggest that HSCs influence macrophage phenotype during acute injury, and that in the absence of HSCs, macrophages may skew toward an antiinflammatory state, supported by the finding that IL-10 levels are higher in HSC-depleted mice treated with carbon tetrachloride and allyl alcohol [3]. While this remains to be fully investigated, additional research in this area could potentially uncover novel therapeutic targets that limit inflammatory liver injury in patients suffering from liver disease.

\section{Conclusion}

Although HSCs were initially described over a century ago, research continues today to understand the mechanisms that regulate HSC activation and to identify the critical functions of these cells after injury. Although great strides have been made in these areas, development of new methods to selectively deplete HSCs or to selectively knockout genes in these cells could lead to important new findings that have the potential to revolutionize treatment of liver disease.

Acknowledgments I thank Kara Kelly, Dominique Garrison, Reynier Urdaneta Moreno, Ryan Albee, and Katherine Roth for critical review of the manuscript. I also sincerely apologize to those whose work I was not able to discuss or reference. There is a tremendous amount of excellent research in the hepatic stellate cell field. Unfortunately, mentioning all of this important work was beyond the scope of this short review

\section{Compliance with Ethics Guidelines}

Conflict of Interest Bryan L. Copple declares no conflict of interest.

Human and Animal Rights and Informed Consent This article does not contain any studies with human or animal subjects performed by any of the authors.

\section{References}

Papers of particular interest, published recently, have been highlighted as:

- Of importance,

•- Of major importance

1. Friedman SL et al (1985) Hepatic lipocytes: the principal collagen-producing cells of normal rat liver. Proc National Acad Sci. 82(24):8681-8685

2. Mederacke I et al (2013) Fate tracing reveals hepatic stellate cells as dominant contributors to liver fibrosis independent of its aetiology. Nat Commun 4:2823. This study demonstrated that hepatic stellate cells are the main collagen producing cell in the liver regardless of the animal model of liver fibrosis

3. • Puche JE et al (2013) A novel murine model to deplete hepatic stellate cells uncovers their role in amplifying liver damage in mice. Hepatology 57(1):339-50. This study was the first to discover a potential role for HSCs in regulation of hepatic injury. This study also determined that hepatic stellate cells are the main collagen producing cell type in the liver during development of fibrosis

4. Friedman SL (2008) Mechanisms of hepatic fibrogenesis. Gastroenterology 134(6): 1655-1669

5. Friedman SL, Arthur MJ (1989) Activation of cultured rat hepatic lipocytes by Kupffer cell conditioned medium. Direct enhancement of matrix synthesis and stimulation of cell proliferation via induction of platelet-derived growth factor receptors. J Clin Invest 84(6): 1780-1785

6. Wong L et al (1994) Induction of beta-platelet-derived growth factor receptor in rat hepatic lipocytes during cellular activation in vivo and in culture. J Clin Invest 94(4):1563-1569

7. Andrae $J$ et al (2008) Role of platelet-derived growth factors in physiology and medicine. Genes Dev 22(10):1276-1312

8. Czochra $P$ et al (2006) Liver fibrosis induced by hepatic overexpression of PDGF-B in transgenic mice. J Hepatol 45(3): $419-428$

9. Thieringer F et al (2008) Spontaneous hepatic fibrosis in transgenic mice overexpressing PDGF-A. Gene 423(1):23-28

10. Borkham-Kamphorst E et al (2007) Pro-fibrogenic potential of PDGF-D in liver fibrosis. J Hepatol 46(6):1064-1074

11. Martin IV et al (2013) Platelet-derived growth factor (PDGF)-C neutralization reveals differential roles of PDGF receptors in liver and kidney fibrosis. Am J Pathol 182(1):107-117

12. Campbell JS et al (2005) Platelet-derived growth factor $C$ induces liver fibrosis, steatosis, and hepatocellular carcinoma. Proc National Acad Sci 102(9):3389-3394

13. Rosenbaum $\mathbf{J}$ et al (1995) Fibroblast growth factor 2 and transforming growth factor beta 1 interactions in human liver myofibroblasts. Gastroenterology 109(6):1986-1996

14. Yu C et al (2003) Role of fibroblast growth factor type 1 and 2 in carbon tetrachloride-induced hepatic injury and fibrogenesis. Am J Pathol 163(4):1653-1662

15. Vu TK et al (1991) Molecular cloning of a functional thrombin receptor reveals a novel proteolytic mechanism of receptor activation. Cell 64(6):1057-1068

16. Gaca MD et al (2002) Regulation of hepatic stellate cell proliferation and collagen synthesis by proteinase-activated receptors. J Hepatol 36(3):362-369

17. Fiorucci S et al (2004) PAR1 antagonism protects against experimental liver fibrosis. Role of proteinase receptors in stellate cell activation. Hepatology 39(2):365-375

18. Rullier A et al (2008) Protease-activated receptor 1 knockout reduces experimentally induced liver fibrosis. Am J Physiol Gastrointest Liver Physiol 294(1):G226-G235

19. Luyendyk JP et al (2011) Fibrinogen deficiency increases liver injury and early growth response-1 (Egr-1) expression in a model of chronic xenobiotic-induced cholestasis. Am J Pathol 178(3): $1117-1125$

20. Cassiman D et al (2001) Human and rat hepatic stellate cells express neurotrophins and neurotrophin receptors. Hepatology 33(1): $148-158$

21. Ruddell RG et al (2006) A role for serotonin (5-HT) in hepatic stellate cell function and liver fibrosis. Am J Pathol 169(3): 861-876

22. Oben JA et al (2003) Norepinephrine and neuropeptide Y promote proliferation and collagen gene expression of hepatic myofibroblastic stellate cells. Biochem Biophys Res Commun 302(4):685-690 
23. Oben JA et al (2004) Hepatic fibrogenesis requires sympathetic neurotransmitters. Gut 53(3):438-445

24. Oben JA et al (2003) Acetylcholine promotes the proliferation and collagen gene expression of myofibroblastic hepatic stellate cells. Biochem Biophys Res Commun 300(1):172-177

25. Blobe GC et al (2000) Role of transforming growth factor beta in human disease. N Engl J Med 342(18):1350-1358

26. Wells RG (2000) Fibrogenesis. V. TGF-beta signaling pathways. Am J Physiol Gastrointest Liver Physiol 279(5):G845-G850

27. Wang B et al (2007) Role of alphavbeta6 integrin in acute biliary fibrosis. Hepatology 46(5):1404-1412

28. Schnabl B et al (2001) The role of Smad3 in mediating mouse hepatic stellate cell activation. Hepatology 34(1):89-100

29. Reimann $\mathrm{T}$ et al (1997) Transforming growth factor-beta1 induces activation of Ras, Raf-1, MEK and MAPK in rat hepatic stellate cells. FEBS Lett 403(1):57-60

30. Hellerbrand $\mathrm{C}$ et al (1999) The role of TGFbeta1 in initiating hepatic stellate cell activation in vivo. J Hepatol 30(1):77-87

31. Kobayashi $\mathrm{H}$ et al (2005) Connective tissue growth factor and progressive fibrosis in biliary atresia. Pediatr Surg Int 21(1):12-16

32. Piao RL et al (2012) Clinical significance of connective tissue growth factor in hepatitis B virus-induced hepatic fibrosis. World J Gastroenterol 18(18):2280-2286

33. Narkewicz MR et al (2005) Connective tissue growth factor expression is increased in biliary epithelial cells in biliary atresia. J pediatr Surg 40(11):1721-1725

34. Abou-Shady $M$ et al (2000) Connective tissue growth factor in human liver cirrhosis. Liver 20(4):296-304

35. Paradis V et al (1999) Expression of connective tissue growth factor in experimental rat and human liver fibrosis. Hepatology 30(4):968-976

36. Paradis V et al (2002) Effects and regulation of connective tissue growth factor on hepatic stellate cells. Lab Invest 82(6):767-774

37. Li G et al (2006) Inhibition of connective tissue growth factor by siRNA prevents liver fibrosis in rats. J Gene Med 8(7):889-900

38. Meng $F$ et al (2012) Interleukin-17 signaling in inflammatory cells, Kupffer, and hepatic stellate cells exacerbates liver fibrosis in mice. Gastroenterology 143(3):765-776

39. Schneiderhan W et al (2001) Oxidized low-density lipoproteins bind to the scavenger receptor, CD36, of hepatic stellate cells and stimulate extracellular matrix synthesis. Hepatology 34(4 Pt 1): 729-737

40. Parola M et al (1996) Induction of procollagen type I gene expression and synthesis in human hepatic stellate cells by 4-hydroxy-2,3-nonenal and other 4-hydroxy-2,3-alkenals is related to their molecular structure. Biochem Biophys Res Commun 222(2):261-264

41. Marra F et al (1999) Monocyte chemotactic protein-1 as a chemoattractant for human hepatic stellate cells. Hepatology 29(1): 140-148

42. Seki E et al (2009) CCR1 and CCR5 promote hepatic fibrosis in mice. J Clin Invest 119(7):1858-1870

43. Seki E et al (2009) CCR2 promotes hepatic fibrosis in mice. Hepatology 50(1):185-197

44. Novo E et al (2007) Proangiogenic cytokines as hypoxia-dependent factors stimulating migration of human hepatic stellate cells. Am J Pathol 170(6):1942-1953

45. Marra F et al (1997) Phosphatidylinositol 3-kinase is required for platelet-derived growth factor's actions on hepatic stellate cells. Gastroenterology 112(4):1297-1306

46. Lee SH et al (2004) Effects and regulation of osteopontin in rat hepatic stellate cells. Biochem Pharm 68(12):2367-2378

47. Urtasun R et al (2012) Osteopontin, an oxidant stress sensitive cytokine, up-regulates collagen-I via integrin alpha(V)beta(3) engagement and $\mathrm{PI} 3 \mathrm{~K} / \mathrm{pAkt} / \mathrm{NFkappaB}$ signaling. Hepatology 55(2):594-608
48. Sahai A et al (2004) Upregulation of osteopontin expression is involved in the development of nonalcoholic steatohepatitis in a dietary murine model. Am J Physiol Gastrointest Liver Physiol 287(1):G264-G273

49. Fickert $\mathrm{P}$ et al (2010) The role of osteopontin and tumor necrosis factor alpha receptor-1 in xenobiotic-induced cholangitis and biliary fibrosis in mice. Lab Invest 90(6):844-852

50. Lorena D et al (2006) Osteopontin expression in normal and fibrotic liver. altered liver healing in osteopontin-deficient mice. J Hepatol 44(2):383-390

51. Coombes JD et al (2014) Osteopontin neutralisation abrogates the liver progenitor cell response and fibrogenesis in mice. Gut In press

52. Thimgan MS, Yee HF Jr (1999) Quantitation of rat hepatic stellate cell contraction: stellate cells' contribution to sinusoidal resistance. Am J Physiol 277(1 Pt 1):G137-G143

53. Kawada $\mathrm{N}$ et al (1992) Eicosanoid-mediated contractility of hepatic stellate cells. Biochem J 285(Pt 2):367-371

54. Rockey DC (1997) New concepts in the pathogenesis of portal hypertension: hepatic wounding and stellate cell contractility. Clin Liver Dis 1(1):13-29

55. Kawada N et al (1993) The contraction of hepatic stellate (Ito) cells stimulated with vasoactive substances. Possible involvement of endothelin 1 and nitric oxide in the regulation of the sinusoidal tonus. Eur J Biochem 213(2):815-823

56. Rockey DC (1995) Characterization of endothelin receptors mediating rat hepatic stellate cell contraction. Biochem Biophys Res Commun 207(2):725-731

57. Pinzani $M$ et al (1996) Endothelin 1 is overexpressed in human cirrhotic liver and exerts multiple effects on activated hepatic stellate cells. Gastroenterology 110(2):534-548

58. Halank M et al (2004) Use of oral endothelin-receptor antagonist bosentan in the treatment of portopulmonary hypertension. Transplantation 77(11):1775-1776

59. Hinterhuber L et al (2004) Endothelin-receptor antagonist treatment of portopulmonary hypertension. Clin Gastroenterol Hepatol 2(11):1039-1042

60. O'Brien KM et al (2013) IL-17A synergistically enhances bile acid-induced inflammation during obstructive cholestasis. Am J Pathol 183(5):1498-1507

61. Dechene A et al (2010) Acute liver failure is associated with elevated liver stiffness and hepatic stellate cell activation. Hepatology 52(3):1008-1016

62. - Nejak-Bowen KN et al (2013) Gliotoxin-induced changes in rat liver regeneration after partial hepatectomy. Liver Int 33(7):1044-55. This study demonstrated a potential role of HSCs in regeneration of the liver after partial hepatectomy

63. Wright MC et al (2001) Gliotoxin stimulates the apoptosis of human and rat hepatic stellate cells and enhances the resolution of liver fibrosis in rats. Gastroenterology 121(3):685-698

64. Anselmi K et al (2007) Gliotoxin causes apoptosis and necrosis of rat Kupffer cells in vitro and in vivo in the absence of oxidative stress: exacerbation by caspase and serine protease inhibition. J Hepatol 47(1):103-113

65. Skrtic $S$ et al (1997) Insulin-like growth factors stimulate expression of hepatocyte growth factor but not transforming growth factor betal in cultured hepatic stellate cells. Endocrinology 138(11):4683-4689

66. Mead JE, Fausto N (1989) Transforming growth factor alpha may be a physiological regulator of liver regeneration by means of an autocrine mechanism. Proc Nat Acad Sci 86(5):1558-1562

67. Evarts RP et al (1992) Expression of transforming growth factoralpha in regenerating liver and during hepatic differentiation. Mol Carcinog 5(1):25-31

68. Zhu NL et al (2012) Hepatic stellate cell-derived delta-like homolog 1 (DLK1) protein in liver regeneration. J Biol Chem 287(13):10355-10367 
69. Wang Y, Sul HS (2006) Ectodomain shedding of preadipocyte factor 1 (Pref-1) by tumor necrosis factor alpha converting enzyme (TACE) and inhibition of adipocyte differentiation. Mol Cell Biol 26(14):5421-5435

70. Taniguchi E et al (2001) Expression and role of vascular endothelial growth factor in liver regeneration after partial hepatectomy in rats. J Histochem Cytochem 49(1):121-130

71. Martinez-Hernandez A, Amenta PS (1995) The extracellular matrix in hepatic regeneration. FASEB J 9(14):1401-1410

72. Shimizu $\mathrm{H}$ et al (2005) Vascular endothelial growth factor and angiopoietins regulate sinusoidal regeneration and remodeling after partial hepatectomy in rats. World J Gastroenterol 11(46): 7254-7260

73. Apte U et al (2009) Enhanced liver regeneration following changes induced by hepatocyte-specific genetic ablation of integrin-linked kinase. Hepatology 50(3):844-851

74. Bissell DM et al (1995) Cell-specific expression of transforming growth factor-beta in rat liver. Evidence for autocrine regulation of hepatocyte proliferation. J Clin Invest 96(1):447-455
75. Watanabe S et al (1998) A novel hepatic stellate (Ito) cell-derived protein, epimorphin, plays a key role in the late stages of liver regeneration. Biochem Biophys Res Comm 250(2):486-490

76. - Stewart RK et al (2014) A novel mouse model of depletion of stellate cells clarifies their role in ischemia/reperfusion- and endotoxin-induced acute liver injury. J Hepatol 60(2):298-305. This study identified a key role for HSCs in inflammatory liver injury after ischemia/reperfusion of the liver and after endotoxin treatment

77. • Chang J et al (2013) Activated hepatic stellate cells mediate the differentiation of macrophages. Hepatol Res 43(6):658-69. This study demonstrated that activated HSCs regulate macrophage polarization

78. Mochizuki A et al (2014) Hepatic stellate cells orchestrate the clearance of necrotic cells in a HIF-1alpha-dependent manner by modulating macrophage phenotype in mice. J Immunol 192:3847-3857 